\title{
InN Nanorods and Epi-layers: Similarities and Differences
}

\author{
Z Liliental-Weber, O.Kryliouk ${ }^{1}$, H.J. Park ${ }^{1}$, J. Mangum ${ }^{1}$, T. Anderson ${ }^{1}$ and W. Schaff ${ }^{2}$ \\ Lawrence Berkeley National Laboratory, Berkeley, CA 94720 m/s 62/203 \\ 1. Dept. of Chemical Engineering, University of Florida, Gainesville, FL 32611, U.S.A. \\ 2. Dept. of Electrical and Computer Engineering, Cornell University, Ithaca, NY
}

Summary: Transmission electron microscopy was applied to study $\mathrm{InN}$ nanorods grown on the a-, c-and r-plane of $\mathrm{Al}_{2} \mathrm{O}_{3}$, and (111) $\mathrm{Si}$ substrates by non-catalytic, template-free hydride metal-organic vapor phase epitaxy (H-MOVPE). Single crystal nanorod growth was obtained on all substrates. However, the shape of the nanorods varied depending on the substrate used. For example, nanorods grown on r-plane sapphire and (111) Si have sharp tips. In contrast, growth on a- and c- planes of $\mathrm{Al}_{2} \mathrm{O}_{3}$ results in flat tips with clear facets on their sides. The structural quality of these nanorods and their growth polarity are compared to crystalline quality, surface roughness, defects and growth polarity of $\mathrm{InN}$ layers grown by $\mathrm{MBE}$ on the same planes of $\mathrm{Al}_{2} \mathrm{O}_{3}$

\section{Introduction}

InN has some promising transport and electronic properties. It has the smallest effective electron mass of all the group-III nitrides, which leads to a potentially high mobility, saturation velocity and a large drift velocity at room temperature. As a result of these properties, there has been rapidly increasing interest in InN for use in optoelectronic devices, such as laser diodes and high efficiency solar cells, as well as high frequency/high power electronic devices. The growth of high quality epitaxial layers of InN has been established by molecular beam epitaxy and metal-organic chemical vapor deposition. However, the fabrication of potential one-dimensional structures, such as nanowires and nanorods, has proven even more difficult since in order to synthesize high quality InN, a very low decomposition temperature and a large pressure of $\mathrm{N}$ is required.

InN nanorods grown on a-, c- and r-planes of $\mathrm{Al}_{2} \mathrm{O}_{3}$ and (111) Si substrates by non-catalytic, template-free hydride metal-organic vapor phase epitaxy (H-MOVPE) and InN layers grown on cand r-plane of $\mathrm{Al}_{2} \mathrm{O}_{3}$ substrates by molecular-beam epitaxy were characterized using Transmission Electron Microscopy (TEM) methods. Structural quality and growth polarity of the nanorods and epilayers were compared.

\section{Experimental}

The InN films were grown by molecular beam epitaxy on c-and r-planes of substrates with a GaN buffer layer. The details of this growth procedure are described elsewhere [1]. The InN nanorods were grown using a horizontal hot wall H-MOVPE reactor. Trimethyl indium (TMIn) and $\mathrm{NH}_{3}$ were used as the In and $\mathrm{N}$ sources, respectively [2]. The growth was performed at atmospheric pressure in an $\mathrm{N}_{2}$ ambient. The growth temperature varied from 400 to $700{ }^{\circ} \mathrm{C} ; \mathrm{HCl} / \mathrm{TMIn}$ inlet mole ratio was varied from 0 to $6 ; \mathrm{V} / \mathrm{III}$ ratio ranged from 100 to $10000 ; \mathrm{N}_{2}$ carrier gas flow rate $=1.6 \mathrm{slm}$. Growth temperature, substrate selection, and $\mathrm{HCl} / \mathrm{TMI}$ and $\mathrm{V} / \mathrm{III}$ ratios controlled the diameter, density and orientation of the nanorods. A JEOL 3010 with an accelerating voltage of $300 \mathrm{keV}$ and a subAngstrom CM 300 were used in these studies. Convergent Beam Electron Diffraction (CBED) along the [1100] zone axis together with computer simulation were used to determine the growth polarity of the layers and some nanorods. Electron Energy Loss Spectroscopy (EELS) in addition to X-ray diffraction (XRD) patterns were used to determine the purity of the nanorods. 


\section{Results and Discussion}

\subsection{Faceting of Nanorods and Roughness of the Layers}

InN nanorods grown on the different planes (a-, c-, and r-) of $\mathrm{Al}_{2} \mathrm{O}_{3}$ and also (111) Si are of a high structural quality, but their shapes and faceting differ from each other. The majority of them are elongated along the wurtzite c-axis, but not all crystals are distributed vertically to the substrate and thus form flower-like or random arrangement. XRD studies showed that the nanorods have a wurtzite structure and the majority of them grow along the [0001] axis. This was also confirmed by Selective Area Diffraction patterns. EELS studies show that only some nanorods might contain a minute concentration of oxygen.

The nanorods grown on the a- and c-planes of $\mathrm{Al}_{2} \mathrm{O}_{3}$ have a hexagonal cross-section and their diameter is in the range of $70-220 \mathrm{~nm}$. They have similar lengths in the range of $700-2000 \mathrm{~nm}$. Usually nanorods grown on the c-plane have slightly smaller diameters $(70-220 \mathrm{~nm})$ compared to those grown on the a-plane $(150-220 \mathrm{~nm})$. Since these nanorods were removed from the substrate for TEM observation they appear rectangular at one end and have small facets at the other end. It is therefore understood that these rectangular shape ends, whose surface is not atomically flat, were earlier attached to the substrate and the ends with facets indicate the growth direction (Fig. 1a).

The nanorods grown on r-plane $\mathrm{Al}_{2} \mathrm{O}_{3}$ are round in cross-section tapering to pencil-shape at the growth front with a small plateau on the c-plane at the tip. Their diameter and length are in the range of 300-600 nm and 1000-2500 nm, respectively. A similar shape of tip was also observed for the nanorods grown on (111) Si (Fig. 1b).
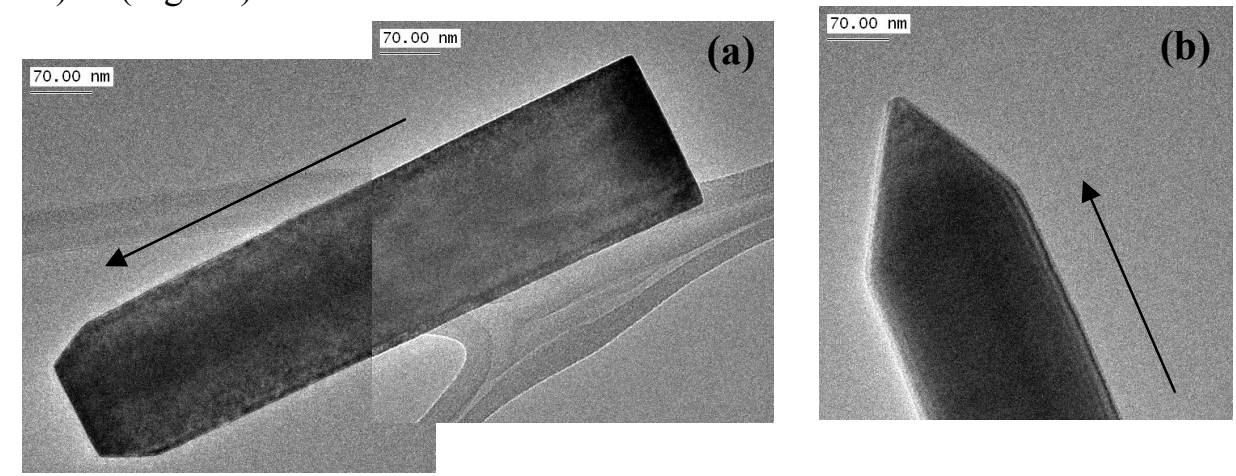

Fig. 1(a) InN nanorod grown on the a-plane and (b) on the r-plane of $\mathrm{Al}_{2} \mathrm{O}_{3}$. Note difference in faceting of their tips and different length of facets on two sides of each crystal. The arrows indicate a growth direction.

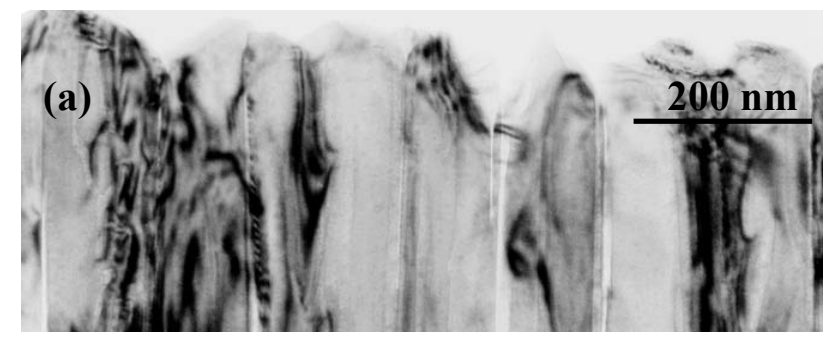
QuickTime $^{\mathrm{TM}}$ and a
TIFF (Uncompressed) decompressor
are needed to see this picture. (b)

\section{$50 \mathrm{~nm}$}

Fig. 2 (a) InN epilayers grown along [1120] direction on the r-plane (1102) $\mathrm{Al}_{2} \mathrm{O}_{3}$. Note columnar growth and strong faceting of all columns; (b) InN layer grown along polar [0002] direction on the cplane (0002) $\mathrm{Al}_{2} \mathrm{O}_{3}$. Much smaller surface roughness and large columnar grains are observed in these layers.

For the differences in shape of faceting of the nanorod tips for growth on the differing substrates, one can find similarities in the surface roughness of InN layers grown on the same surfaces of sapphire. Independently from the substrate surface on which epi-layers were grown, columnar growth was observed. These columns are more pronounced in the layers grown in non-polar orientation (grown 
on r-plane of $\mathrm{Al}_{2} \mathrm{O}_{3}$ ) where occasionally separation between the columns is visible (Fig. 2a). Much stronger faceting (on the same planes as for the nanorods) is observed at the sample surface grown on the r-plane of $\mathrm{Al}_{2} \mathrm{O}_{3}$. In addition, the surface roughness can be as large as $130 \mathrm{~nm}$, while for the layers grown on the c-plane of $\mathrm{Al}_{2} \mathrm{O}_{3}$ the roughness does not exceed 10-20 nm (Fig. 2b).

\subsection{Growth Polarity of the InN Epi-layers and Nanorods}

Convergent Beam Electron Diffraction (CBED) was applied to study the growth polarity of InN layers grown on the c-plane of sapphire and $\mathrm{InN}$ nanorods grown also on the c-plane of the same substrate using a JEOL 3010 TEM. These patterns were taken for different sample thicknesses. CBED patterns for the same zone axis and sample thickness were simulated for the accelerating voltage $(300 \mathrm{keV})$, as used in the experiment. A good agreement between experimental and calculated patterns was obtained. Based on these experiments and taking into account the rotation angle between the image and a diffraction pattern in our microscope it was determined that the layers were grown with In polarity but the studied nanorods grown on c-plane of $\mathrm{Al}_{2} \mathrm{O}_{3}$ were grown with N-polarity. Since the statistics for polarity determination of nanorods are rather poor, it is very difficult to make more general statement as to if the nanocrystals grown on different surfaces of sapphire have a similar polarity.
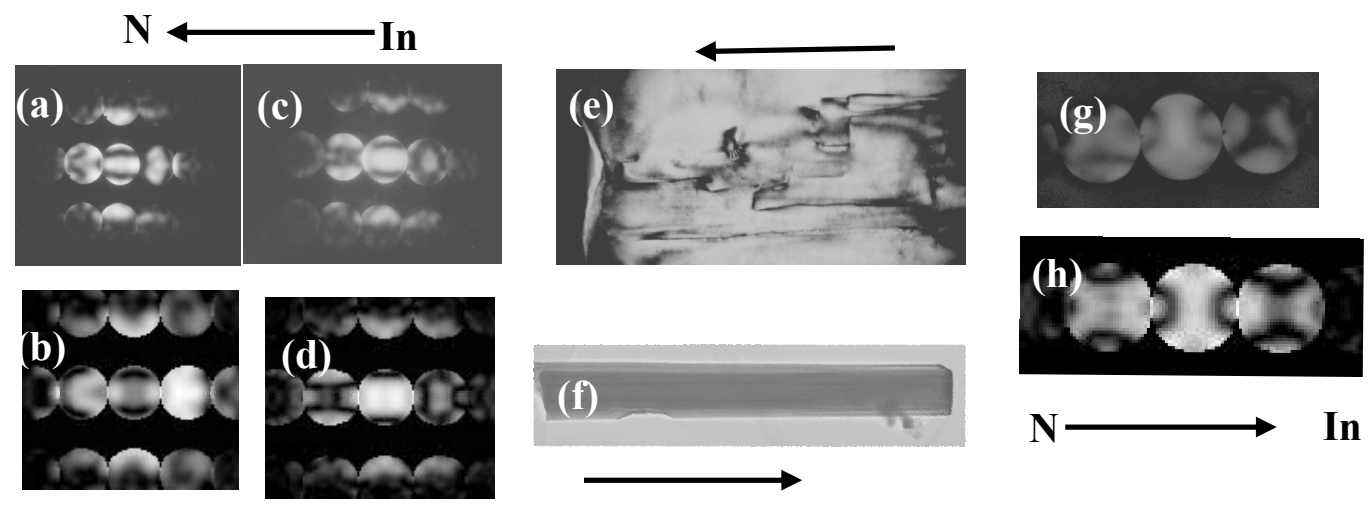

Fig. 3 (a-b) and (c-d) Pairs of experimental and calculated CBED patterns for InN with the thickness of $65 \mathrm{~nm}$ and $100 \mathrm{~nm}$ with an indication of In and $\mathrm{N}$ atom arrangement along c-axis confirming In growth polarity of the layer; (e) InN epilayer with growth direction indicated by arrow, (f) InN nanorod with growth direction indicated, $(\mathrm{g}-\mathrm{h})$ a pair of experimental and calculated CBED patterns showing $\mathrm{N}$ growth polarity of the nanorod.

\subsection{Defects in the InN Epi-layers and Nanorods}

The main defects in the InN layers grown on the c-plane of $\mathrm{Al}_{2} \mathrm{O}_{3}$ are threading dislocations, which propagate along the growth direction. Their density is in the range of $8 \times 10^{9} \mathrm{~cm}^{-2}-2 \times 10^{10} \mathrm{~cm}^{-2}$. This is only slightly larger than the density of dislocations in the underlying $\mathrm{GaN}$ buffer layer. Basal stacking faults (BSFs) formed on c-planes are also observed. For the samples grown in the non-polar direction, prismatic stacking faults (PSFs) are observed in addition to dislocations and BSFs.

InN nanorods show high crystalline perfection with very clean and abrupt side walls (Fig. 4a). However, occasionally the presence of twins can be observed leading to corrugated side walls (Fig. $4 b$ ). As mentioned at the beginning of this paper, faceting of the nanorod tips is observed. These facets have different lengths on the opposite sides of the nanorods. Usually the longer facet is abrupt (Fig. 4c), but along a shorter facet twins are also observed (Fig. 4d-e). Twins were not observed in the epi-layers.

In some nanocrystals grown on the a-plane of $\mathrm{Al}_{2} \mathrm{O}_{3}$ the diameter is slightly changing along the length and a v-shape grove appears along one elongated side of the crystal. CBED patterns show two

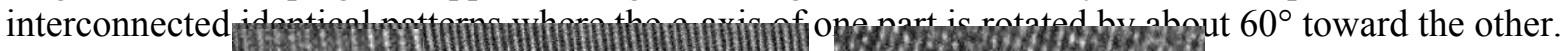
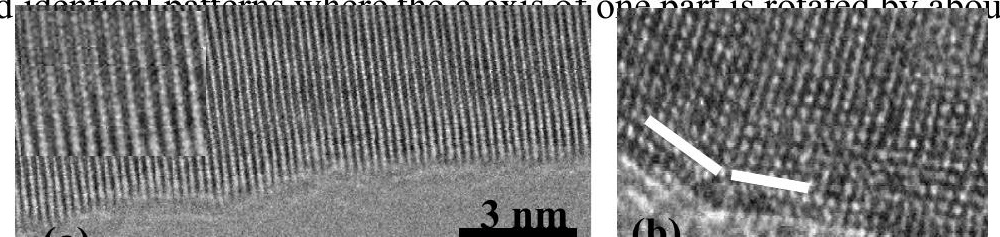


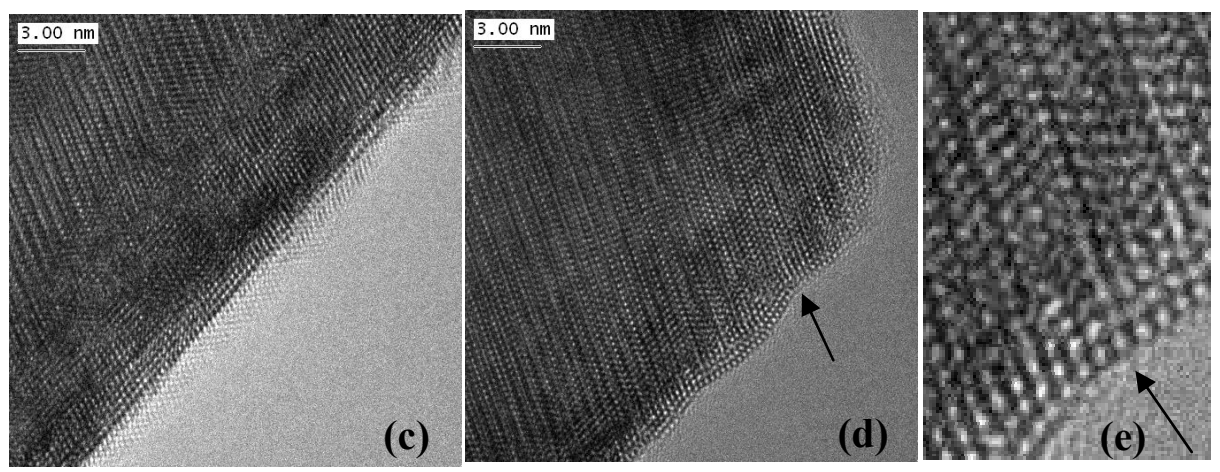

Fig. 4 (a) Side wall of a nanorod showing high perfection, (b) a similar side wall of a nanocrystal grown on a-plane showing corrugated walls with twins, (c-d) high resolution images of a long and short facet of a tip of a nanorod grown on Si substrates, (e) a magnified area of the tip facet showing formation of a twin

This coincides with the [0111] direction, which has the same displacement vector as a prismatic stacking fault (PSF). This suggests that PSFs might be present in these nanorods (similarly to the InN MBE grown layers). PSFs may give rise to the growth of the crystals at different angles and thus the "flower-like" features.

\section{Conclusions}

These studies show that InN epi-layers grown on c- and r-plane of $\mathrm{Al}_{2} \mathrm{O}_{3}$ have different surface roughness. Much more narrow columns with strong faceting are observed for the growth in non-polar direction. This coincides with the different shape of the tips of the nanorods grown on different planes of sapphire.

The majority of nanocrystals have high crystalline perfection but in some of them twinning was observed along the sidewalls and tips of the nanorods. However, this type of defect was not observed in the epilayers. The majority of defects present in the latest material are dislocations and stacking faults. Particularly, basal and prismatic stacking faults are observed in the layers grown on non-polar surfaces. Based on CBED studies of nanorods grown on the a-plane $\mathrm{Al}_{2} \mathrm{O}_{3}$, prismatic stacking faults might be present in the nanorods grown on the same substrate since the two patterns are rotated by about $60^{\circ}$ to each other. This coincides with the angle between the c-axis and [0111] direction, which has the same displacement vector as a prismatic stacking fault.

\section{Acknowledgment}

This work is supported by the U.S. Department of Energy under Contract No. DE-AC02-05CH11231. The work at UF is partially supported by NSF (CTS-031178) and NASA Kennedy Space Center Grant NAG 10-316, ONR (N00014-98-1-04) and NSF DMR 0400416.

\section{References}

1. Lu H, Schaff W J, Hwang J, Wu H et al 2000 Appl. Phys. Lett. 77, 2548

2. Kryliouk O, Reed M, Dann T, Anderson T, and Chai B 1999 Mater. Sci. Eng. B 59, 6. 